
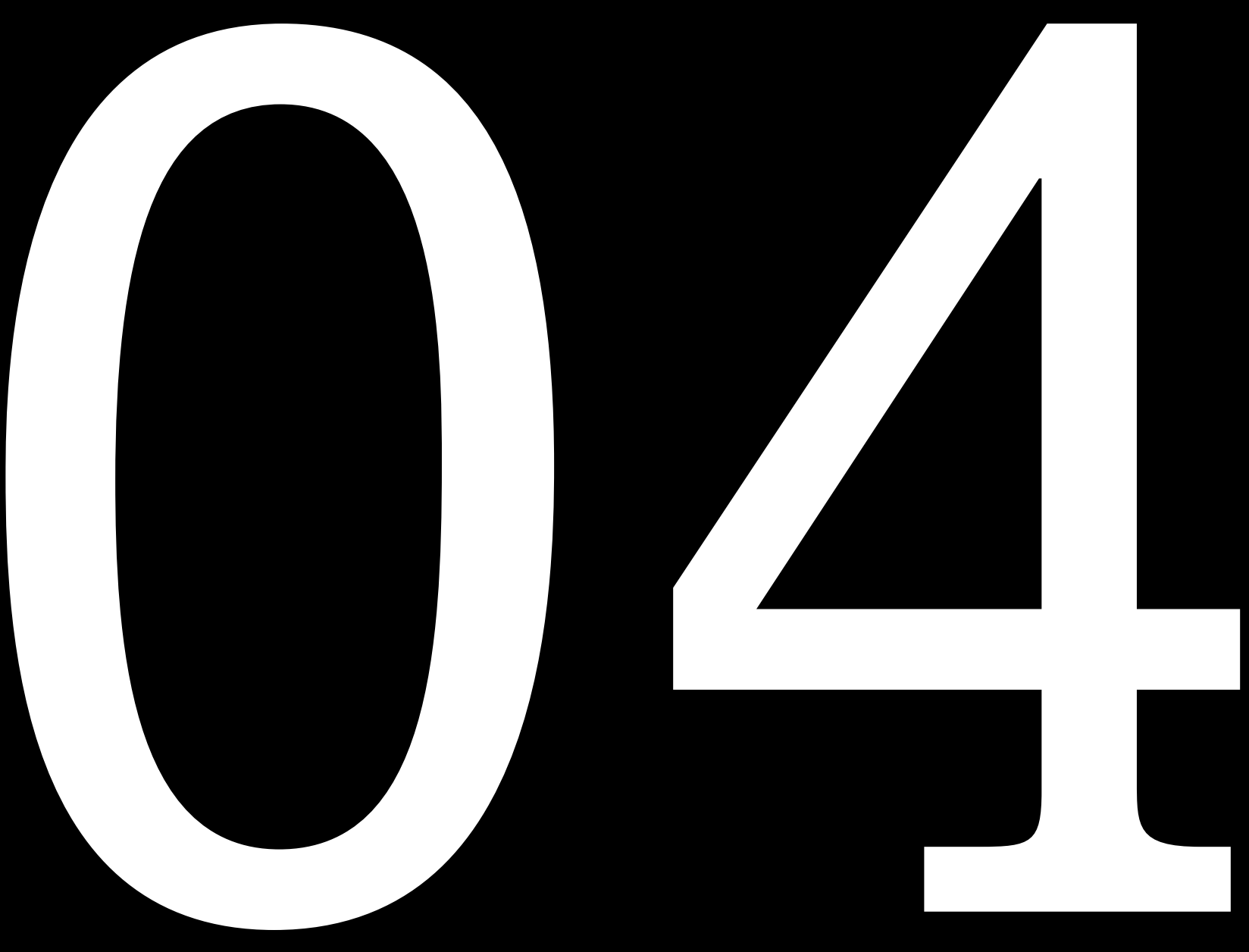

DOI: https://doi.org/10.14483/2422278X.15951 


\section{Los imaginarios de paz: construcción colectiva de estudiantes $y$ maestros ${ }^{1}$}

\section{The imaginary of peace: student and teacher collective construction}

\section{Luz Stella Fuentes Fuentes ${ }^{2}$}

Para citar este artículo: Fuentes, L. (2020). Los imaginarios de paz: construcción colectiva de estudiantes y maestros. Ciudad Paz-ando, 13(1), pp. 45-53.

Fecha de recepción: 27 de mayo de 2019

Fecha de aprobación: 27 de junio de 2020

\footnotetext{
1 El presente artículo hace parte de la investigación titulada: Los imaginarios de paz construcción colectiva de estudiantes y maestros. La cual, a partir de la pregunta sobre los imaginarios de paz presentes en estudiante y maestros, y partiendo de postulados teóricos de autores como Chapela (2013), Cengelci y Gurdogan (2016) y Erreguerena (2002) entre otros. Se desarrollo una investigación desde una perspectiva cualitativa, con enfoque hermenéutico, en la cual participaron dieciocho estudiantes y nueve maestros de educación básica secundaria, con los cuales se realizaron entrevistas de grupo focal. El análisis de la información se fundamentó en el análisis de contenido. Los resultados obtenidos permitieron concluir que existe un vínculo indeleble entre convivencia y paz, los estudiantes se orientan a un concepto de paz como estado anhelado, vivencia en armonía, libertad y justicia, mientras que los maestros lo precisan como un proceso de construcción y responsabilidad social y sitúan el diálogo como dimensión de la misma. Respecto al proceso de paz, los maestros resaltan el esfuerzo loable del Estado colombiano y la reglamentación del Sistema Integral de Verdad, Justicia, Reparación y No Repetición. Inicio: abril 2017, fin: junio 2018 duración.

2 Docente de educación básica secundaria y universitaria. Pertenece al grupo de investigación REDINA de la Corporación Universitaria del Caribe CECAR en Sincelejo. Orienta una cátedra en la Facultad de Humanidades y Educación. Correo electrónico: luz.fuentes@cecar.edu.co,
} 
Partiendo de la pregunta sobre los imaginarios de paz en estudiantes y maestros de educación básica secundaria, se presenta aquí el resultado de entrevistas a grupos focales relacionada con el concepto y el proceso de paz. La unidad de análisis involucra grupos de jóvenes y maestros (dieciocho estudiantes distribuidos en tres grupos de seis y nueve maestros distribuidos en dos grupos). El enfoque cualitativo naturalista e interpretativo y el análisis descriptivo-interpretativo. Se concluyó que existe un vínculo indeleble entre convivencia y paz, los estudiantes se orientan a un concepto de paz como estado anhelado, vivencia en armonía, libertad y justicia, mientras que los maestros lo precisan como un proceso de construcción y responsabilidad social y sitúan el diálogo como dimensión de la misma. Respecto al proceso de paz, los maestros resaltan el esfuerzo loable del Estado colombiano y la reglamentación del Sistema Integral de Verdad, Justicia, Reparación y No Repetición.

Palabras clave: convivencia, estudiante de secundaria, imaginario, paz.

\section{ABSTRACT}

Starting from the question about peace imaginaries between basic secondary students and teachers, here is presented the result of focus group interviews related to the concept and the peace process. The unit of analysis involves young and teacher groups (eighteen students distributed on three six person groups and nine teachers distributed on two groups). The naturalist and interpretative qualitative focus and descriptive-interpretative analysis. It's been concluded that there is an invisible link between coexistence and peace, students are oriented to a peace concept as longed-for status, experience in harmony, freedom and justice, meanwhile, teachers precise it as a construction and social responsibility process and they place the dialogue as a dimension of it. On respect to the peace process, teachers highlight the laudable effort of Colombian state and the Integral system of truth, justice, repair and not repeating regulation.

Keywords: coexistence, high school student, imaginary, peace. 


\section{Introducción}

Este artículo es el resultado parcial de los hallazgos encontrados en un proyecto sobre el arte como medio de expresión de los imaginarios de paz. En este aparte se exploran las voces de jóvenes y maestros relacionadas con la concepción de paz y el proceso adelantado por el Gobierno colombiano.

Hablar de paz en tiempos de guerra es difícil, pero más difícil es hablar de paz en procesos de paz cuando no cesan los comportamientos asociados a la guerra. Hoy, Colombia vive momentos históricos coyunturales: proceso de paz y preocupación e incertidumbre por la continuación de los acuerdos de paz ante un nuevo presidente. La guerra, como guerra después de los acuerdos de paz, no ha terminado por completo, aún se mantienen rasgos de ella que hacen florecer en los ciudadanos la inseguridad y el miedo. Un estudiante entrevistado expresa: "Miedo a todo", "Miedo a no poder salir a la calle en libertad" (Comunicación personal, Jiménez, 2017).

El clima de paz que respira Colombia se ve muchas veces nublado por las acciones bélicas de las personas que no están de acuerdo con una paz duradera y real. Todo ciudadano adulto, joven o niño, untado de ambientes de paz tiene sus percepciones acerca de ella, pero se consultan muy poco. No es lo mismo leer sobre un proceso de guerra a que se vivencie, puesto que se habla con certeza, solo de aquello que se experimenta. De igual forma, no es igual leer sobre la paz a que se empiece a vivir desde los albores de un proceso direccionado para su consecución. Todos estos sentimientos se evidencian en las intervenciones de los sujetos implicados en esta investigación, estudiantes de básica y docentes.

Mediante este escrito se comparte una mirada de aquellos ciudadanos poco preguntados acerca de la paz y de los procesos adelantados en el país, son ellos jóvenes y maestros de educación básica secundaria quienes en este ejercicio fueron abordados en una entrevista y conceptualizaron acerca de la paz y del proceso que se vive en Colombia. Fue necesario ubicar las categorías convencionales paz e imaginarios, pero luego fueron emergiendo nuevas categorías producto de las entrevistas realizadas. Se consultaron a diecisiete estudiantes y nueve maestros; luego, se sistematizó la información y mediante análisis de contenido se codificó. Desde la interpretación emergieron categorías con las cuales se construyó la teoría emergente: un concepto de paz producto de los imaginarios que poseen los estudiantes y maestros.

En la búsqueda de la literatura se encontró elaboración conceptual sobre la paz de la Unesco (1995; 2011), Organización de las Naciones Unidas (ONU) y la Unicef, además de investigadores como: Cengelci y Gurdogan (2016), Fuentes (2018), Erreguerena (2002), Chapela (2013), Aigneren (2002), entre otros.

Actualmente, la paz se constituye como uno de los asuntos en la época actual que requieren ser atendidos de forma prioritaria, pues Colombia es un país donde se habla de paz y se sigue matando; donde la paz es un derecho constitucional y, al tiempo, un valor que hay que cultivar en las personas, siendo la familia y la escuela instituciones a las que se les demanda un alto compromiso en su enseñanza y divulgación.

Este estudio se considera relevante por las condiciones socioculturales presentes en los sujetos entrevistados, la realidad de una escuela afectada por la violencia presente en las familias y los mensajes de la televisión, lo cual recrea la vida de todos aquellos que han causado daño al país y precisamente se constituye en la actividad donde los ciudadanos del pueblo invierten su tiempo libre, disfrutando los programas cuyo contenido está impregnado de lo mismo que les ha producido daño y dolor.

Antes de iniciar a hablar acerca de la paz, es necesario sentar unas bases teóricas sobre el término imaginario. Castoriadis (1993), citado en Erreguerena (2002), plantea que:

Un imaginario social es una construcción socio histórica que abarca el conjunto de instituciones, normas y símbolos que comparte un determinado grupo social y, que, pese a su carácter imaginado, opera en la realidad ofreciendo tanto oportunidades como restricciones para el accionar del sujeto. (p. 40)

Es inevitable el carácter histórico que tiene el imaginario, puesto que se construye en momentos específicos de la realidad vivida por los ciudadanos y esta connotación hace que el imaginario se cree y recree de forma constante, no sea estático, ya que hoy se tiene construido un concepto sobre la realidad y en otro momento histórico puede estar renovado. Ello se corrobora cuando se afirma: "el imaginario social es una capacidad imaginante, un orden de sentido, una producción de significaciones colectivas que al ser producida se va transformando [...] y mediante el imaginario social sabemos quiénes somos y qué papel debemos desempeñar en la sociedad" (Franco, 1999, citado en Erreguerena, 2002, p. 40).

Lo anterior implica que cada ciudadano innova sobre las ideas que tiene acerca de sí mismo, su misión y espacio que ocupa en la comunidad o localidad. Ahora bien, "los imaginarios sociales se fortalecen de una base de conocimientos y prácticas dadas en la otredad, además, se fortalecen de la capacidad cognitiva de imaginar y recrear lo vivido" (Fuentes, 2018).

Asimismo, sobre la paz se han construido varios conceptos y para este apartado se retoma a Chapela (2013), en la caja de herramientas en educación para la paz de la Unesco, quien presenta el concepto de paz asociado a:

Ausencia de conflictos bélicos, la presencia de condiciones para que personas, grupos y comunidades consigan identificar, cultivar, desarrollar y realizar sus potencias, al convertirlas en actos reales que incidan en 
el mejoramiento de ellos mismos y de su entorno, en el fortalecimiento de sus proyectos y en la realización de sus legítimas aspiraciones ecológicas, económicas, culturales, políticas y sociales. La paz es una aspiración que nos invita a trabajar en la construcción de condiciones que la hagan posible. (Chapela, 2013, p. 13)

Este concepto coincide con algunos aspectos relacionados en los imaginarios de paz tanto de maestros como de los estudiantes, los cuales surgieron en las entrevistas realizadas. Del mismo modo, Chapela (2013) expresa:

Que la paz invita a trabajar cada día para construirla, para alcanzarla. Por eso cuando se vive en una cultura de paz, las personas y los grupos, tanto los gobiernos y las instituciones, logran ser cada día mejores y consiguen organizar sus recursos (ecológicos, económicos, políticos y culturales) de la mejor manera, para alcanzar por lo menos, los máximos logros posibles. (pp. 19-20)

En esta línea, la ONU, citada en Fuentes (2018), define la cultura de paz como un conjunto de valores, actitudes, comportamientos y estilos de vida que rechazan la violencia y previenen los conflictos atacando a sus raíces a través del diálogo y la negociación entre los individuos, los grupos y los estados.

Para efectos de esta investigación, se presenta una conceptualización de paz desde las vivencias de los estudiantes, en primera medida, y, posteriormente, los docentes. El concepto de paz construido desde las expresiones de estudiantes hace referencia a la paz como estado ideal, el cual puede asumir una persona o grupo social. Un estado anhelado, pues la sociedad posee esperanzas de una ausencia de violencia. Asimismo, la paz es producto de una educación fundada en valores, cimentada en acciones de convivencia, donde emana la armonía, el buen trato, la libre expresión y la seguridad; así, para ser alcanzada se requiere de un mutuo acuerdo entre las partes implicadas en el conflicto. Desde este concepto se logran evidenciar categorías relevantes que en el análisis se consideran.

En la misma línea, el concepto de paz elaborado por los docentes se ubica en el conjunto de condiciones mínimas que deben existir en las relaciones de un grupo social, manifestadas en estado del alma, estado de bienestar, justicia social, equidad, reparación a víctimas, empoderamiento, paz como proceso en construcción, equilibrio emocional y responsabilidad social.

Por consiguiente, es indudable que los impactos psicológicos de la violencia y el trauma que ocasiona el posconflicto se reflejan en los entornos escolares. Esta realidad hizo pensar en preguntar a los jóvenes sobre la paz, cómo la entienden y perciben. Así, la búsqueda de información corrobora que existen pocos estudios que involucren a los niños y jóvenes a hablar con otros pares y con adultos sobre la paz, específicamente con docentes.

\section{Metodología}

El enfoque de esta investigación es hermenéutico. Tiene como propósito describir e interpretar un texto para dar sentido al escrito, es decir, busca dar cuenta de la intención implícita en el texto o mensaje y que se muestra en las marcas textuales expresadas por parte de los participantes (para este caso particular los estudiantes y docentes). En el análisis hermenéutico ocurre un dato curioso, según Palmer (2002): "la experiencia hermenéutica es una forma subjetiva de objetividad" (p. 299).

Esta investigación involucra pequeños grupos focales de jóvenes de educación básica secundaria, dieciocho estudiantes en total, y grupos focales de nueve docentes, con los cuales se conversó sobre la paz y el proceso de paz. En las entrevistas realizadas se emplearon preguntas abiertas. Con los jóvenes se organizaron tres grupos de seis estudiantes a quienes se les hizo la misma pregunta: “¿De qué manera definen la paz?”. Por otro lado, con los docentes, se emplea igual metodología en la entrevista de grupo focal, pues se les solicitó responder la misma pregunta que se aplicó a los jóvenes, pero se adiciona una diferente: su opinión acerca del proceso de paz.

Es importante precisar que el grupo focal es una modalidad de entrevista grupal, abierta y estructurada que toma la forma de una conversación, donde el investigador formula preguntas sobre un tópico específico. Tiene como propósito "registrar cómo los participantes elaboran grupalmente su realidad y experiencia" (Aigneren, 2002). Asimismo, el grupo focal se constituye en la oportunidad para promover la conversación y la interacción (Freeman, 2013).

Para este caso, el empleo del grupo focal es utilizado para dar cumplimiento al objetivo inicial: describir cómo los grupos involucrados dan cuenta de los imaginarios de paz en los contenidos textuales de sus expresiones, concibiendo que todo acto comunicativo se enmarca en un contexto sociocultural y, a partir de allí, se enfatiza en la comprensión de los significados. De hecho, los momentos cruciales que vive el país sirven de base para entender la concepción de paz actual en estudiantes y docentes, lo que provoca esta reflexión.

\section{Análisis y discusión}

El análisis de la información se desarrolló desde un enfoque hermenéutico, por lo que se empleó el análisis de contenido. Una vez recogida la información, se sistematiza y codifica desde categorías como pensamientos o ideas de paz y percepciones del proceso de paz en Colombia, esta última en la conversación de los docentes. Desde allí, surgen códigos relevantes que se referencian a continuación.

Un primer aspecto encontrado en los imaginarios de paz de los estudiantes está relacionado con la paz como estado ideal, "ausencia de guerras, conflictos y lucha" (Comunicación personal, Márquez, 2017). "Paz es poder salir de tu casa y que no tengas miedo o temor de que te pase 
algo" (Comunicación personal, Jiménez, 2017). En este sentido, Cengelci y Gurdogan (2016), sostienen que:

Las sociedades exigen que sus ciudadanos, sean respetuosos con los demás, se comprendan, contribuyan a la producción de la sociedad y protejan los valores culturales, por consiguiente, las personas necesitan desarrollar valores como la paz, el amor, el respeto, la tolerancia, la igualdad, y las habilidades como empatía, comunicación efectiva, resolución de conflictos, cooperación y resolución de problemas.

Para ello, es necesario que se enseñe a vivir en paz, constituyéndose la paz en un derecho humano y un valor posible de enseñar y aprender. Como valor, la paz debe guiar las relaciones familiares, escolares y comunitarias, además de ser visible en los comportamientos de la vida cotidiana, específicamente en la manera como asumen los conflictos que se presentan diariamente. Frente a ello, surge de las entrevistas que "la paz es tranquilidad, ausencia de violencia en seres humanas" (Comunicación personal, Luna, 2017). "Es aquello con lo que el ser humano no ha logrado vivir, por falta de educación” (Comunicación personal, Herazo, 2017). Para tal efecto, en el alto compromiso que le compete, el Estado colombiano requiere no solo reglamentar normas, sino capacitar en las mismas a través de diferentes medios y monitorear su aprehensión para que puedan ser interiorizadas por los ciudadanos. Aunque educar para la paz es una tarea obligada de la escuela y la familia, ambas necesitan herramientas para consolidarla.

En consecuencia, no es suficiente para resolver el problema social un proyecto de derechos humanos y de valores establecido como obligatorio para su desarrollo en la escuela si las actividades que se realizan están desligadas de la vida de la comunidad educativa y, además, se desarrollan de forma aislada, sin una secuencia didáctica que contenga la evaluación correspondiente de la regulación del aprendizaje. Lo anterior tiene más implicaciones cuando el currículo oculto se constituye en más poderoso, mostrando comportamientos en la comunidad educativa contrarios a lo que se divulga. De tal forma que a instituciones como la familia y la escuela les corresponde jerarquizar valores y ser consecuentes con estos en sus vivencias desde el marco de los derechos humanos.

Como derecho humano todas las personas, grupos sociales, localidades y países son herederos de la paz, puesto que se tiene derecho a vivir en paz y armonía, y gozar de las libertades constitucionales. En este sentido, se nace en paz, vivimos en paz y morimos en paz. Surge de las entrevistas: "Con la paz seremos felices todos y para la paz tenemos que poner de nuestra parte" (Comunicación personal, Romero, 2017). En esta línea, la paz se quiere, exige y se demanda al Estado. Para este caso, el Estado colombiano la decreta el artículo 22 de la Constitución Política de 1991 y la reglamenta desde el Acto Legislativo
01 del 4 de abril de 2017, por medio del cual se crea un título transitorio de las normas para la terminación del conflicto armado y la construcción de una paz estable y duradera (art. 1). Lo anterior se materializa en el Sistema Integral de Verdad, Justicia, Reparación y No Repetición.

Al considerar los participantes la paz como esperanza, se distingue la añoranza en el corazón de las personas, pues es indiscutible que la paz es "un requisito para la supervivencia de los humanos, y las generaciones, y el desarrollo de las naciones" (Baltork, Manssoori y Azad, 2015, p. 33). La mayoría de los entrevistados tuvieron la oportunidad de vivir en lugares tranquilos que en los momentos de guerra se volvieron inaguantables, perturbando la calidad de vida de los habitantes. Surge en las entrevistas que: "La paz es la esperanza de todos los ciudadanos” (Comunicación personal, Romero, 2017). Por tanto, la calidad de vida de los pueblos que han vivido episodios de guerra constante o han permanecido en ella durante largo tiempo se han afectado tanto en lo biológico como en lo sociológico y psicológico, puesto que, conforme a la tradición, un cuerpo sano proporciona una mente sana y viceversa.

De igual forma, se logra evidenciar en los imaginarios de paz la dimensión negativa de ella, relacionada con ausencia de conflicto, esto connota ausencias de guerra, ausencia de actividades bélicas o una disminución de conflicto (Galtung, 1969; 1996 citado en Sagkal, Turnuklu y Totan, 2012). Surge en las entrevistas que: "Paz es lo que todos anhelamos, pero pocos logramos" (Comunicación personal, Álvarez, 2017). Sin embargo, es importante destacar que existe una dimensión positiva de la paz y ocurre cuando se resuelven los conflictos desde acuerdos, pero con presencia de justicia social, puesto que una ausencia de conflicto no garantiza que haya justicia social.

Los problemas que enfrenta la sociedad actualmente, como conflictos de poder entre grupos, guerras y enfermedades, solo se pueden solucionar si existe una preocupación y compromiso generalizado de toda la sociedad por un trabajo fundamentado en valores como el amor, el respeto, la tolerancia, la cooperación humana, la empatía y la paz. La paz es la concreción de todos los valores humanos; por esto, se requiere una educación fundamentada en valores para poder así educar en la paz y para la paz. En consecuencia, la paz al ser un concepto positivo abarca el derecho a ser educado.

Actualmente, el currículo de las escuelas colombianas contiene la Cátedra de la Paz con la intención de buscar el desarrollo de una comprensión positiva de la paz. Para ello, la escuela y la universidad, en su prolongación, requieren empoderarse de este rol y coparticipar de una serie de actividades que conduzcan a la adquisición de comportamientos proactivos para una sana convivencia. De esta forma, la generación venidera tendrá la posibilidad de sentir al otro como su prójimo, como su extensión, como el tú que soy yo. Un estudiante expresa: "la paz 
es el mayor progreso para cualquier pueblo o nación del mundo" (Comunicación personal, Contreras, 2017). Se añade a este derecho la seguridad humana y a vivir en un ambiente que inspire confianza y sea saludable.

Asimismo, se encuentra presente en esta concepción de paz la convivencia como interacción intrapersonal e interpersonal, la cual se fundamenta en el buen trato, la armonía, la buena comunicación manifestada en la libre expresión y la seguridad social. Un estudiante dice: "Es un valor que identifica la armonía, la solidaridad" (Comunicación personal, Hernández, 2017). Esto devela una paz positiva, donde la injusticia social no tiene presencia y la violencia estructural se quebranta para dar paso a los escenarios de tranquilidad y libertad en todas las manifestaciones. Erkman (2012), citado en Cengelci y Gurdogan (2016), incluye "la armonía de las personas entre sí y la naturaleza, lidiando con las diferencias y compartiendo recursos en la justicia" (p. 183).

Se constituye como una urgencia nacional el desarrollar una cultura de paz en los establecimientos educativos colombianos, lo anterior desde la implementación de programas concebidos como procesos de formación en valores y desarrollo de habilidades como la empatía y resolución de conflicto. La educación para la paz debe inspirar una visión autentica y efectiva en los estudiantes. Siguiendo a Cengelci y Gurdogan (2016) es posible afirmar que la escuela puede facilitar las condiciones y los recursos necesarios para una educación para la paz. Corresponde al Ministerio de Educación Nacional y, en su efecto, a las secretarias de educación municipal y departamental, asumir desde la gestión e inversión planes estratégicos y líneas de acción definidas.

Hoy por hoy, la convivencia se constituye en una prioridad desde la escuela, pues todos los conflictos que se vislumbran en ella son el efecto de lo que se vive afuera de los muros de los establecimientos educativos, es decir, la escuela está permeada por todo lo que ocurre en el exterior. Convivir es una necesidad y está asociada con la relación que se establece con el otro en el marco del respeto y tolerancia, pero desde la diversidad. Según Fuentes, Pérez y Medrano (2018) conciben la convivencia como "un conjunto de experiencias, armónicas o no, que precisan la relación entre las personas y los diferentes grupos a los que pertenecen" (p. 239). Se corrobora que la convivencia puede ser positiva o negativa. En la primera están presentes los acuerdos, reglas y normas y en la segunda hay ausencia de estas, o se encuentran tácitas y muchas veces concertadas, pero no interiorizadas.

En el posconflicto, entre los organismos internacionales y los nacionales urge una educación para la paz. La Unicef, citada en Ávila (2012), define la educación para la paz como:

Un proceso de promoción del conocimiento, las capacidades, las actitudes y los valores necesarios para pro- ducir cambios de comportamiento que permitan a los niños, los jóvenes y los adultos prevenir los conflictos y la violencia, tanto la violencia evidente como la estructural; resolver conflictos de manera pacífica; y crear condiciones que conduzcan a la paz, tanto a escala interpersonal, como intergrupal, nacional o internacional. (pp. 186-187)

A continuación, se relacionan elementos de opinión retomados en el grupo focal de los maestros, en quienes fue reiterado considerar la paz como proceso de construcción, detectada claramente en expresiones como: "la paz se debe construir con todos los colectivos sociales" (Comunicación personal, Lora, 2017). Para ello, sugieren la democracia como estrategia de participación ciudadana apropiada para que la paz se construya desde el "respeto a las reglas, vía libre a los valores y dignidad humana" (Comunicación personal, Lora, 2017). De igual manera, aflora la categoría de responsabilidad social desde las voces de maestros: "la paz es una responsabilidad social, de cada ser humano, la cual se construye día a día con nuestros actos; y se fundamenta en la disciplina y el empoderamiento de cada labor que se realiza en todos los contextos" (Comunicación personal, Galindo, 2017). Se logra entrever la necesidad de asumir la paz como compromiso de todos los ciudadanos y no de los vinculados directamente en el proceso; a su vez, se evidencia la ausencia de ese compromiso por parte del ciudadano. Siendo así, la escuela y la familia necesita fortalecer aún más la formación en la ciudadanía y en los valores humanos.

Otro maestro expresó que "hay indiferencia en la población por el proceso de paz, queremos ser solo espectadores y criticar, mas no ser protagonistas de esta conquista", concluye: "tenemos el balón de la historia a nuestro alcance y debemos anotarle un gol a la indiferencia" (Comunicación personal, Lora, 2017). Por tanto, se manifiesta una combinación entre esperanza y desesperanza, credulidad e incredulidad, en lo que observan y evidencian se vislumbra en el corolario el balón y el gol, significando la oportunidad que se tiene de hacer apuesta en la historia de un proceso de paz con participación de todos.

En este sentido, se vislumbra importante fortalecer, aprender y desarrollar equilibrio emocional, categoría que surge en varios entrevistados, quienes aducen que este ayuda a actuar de forma coherente y con dominio propio. Asimismo, corresponde a la categoría estado de bienestar y declarada en otros como transformación individual que afecta a una colectividad, al expresar: "la paz nace en el corazón del hombre", "la paz conduce a una actuación con sabiduría y discernimiento" (Comunicación personal, Monterroza, 2017). Se logra entrever dos dones del espíritu declarados en la Santa Biblia como dones de conocimiento, esto implica que hace falta apropiarse más del proceso de paz, de la historia de la guerra, sus causas, 
sus consecuencias y, sobre todo, atender la reparación a víctimas, las cuales han recibido una atención psicosocial mediana, diluida muchas veces en las contrataciones estatales y en las transferencias de organismos internacionales. Toda unidad de víctima debe corresponder con atención integral a las víctimas del conflicto (directas e indirectas); de esta manera, dice una entrevistada: "ejemplo, si una familia se desplaza y llega donde un hijo que vive en otro pueblo, este debe ser atendido también como víctima indirecta porque está recibiendo una carga emocional y social que antes de la llegada no tenía" (Comunicación personal, Lastre, 2017).

Aunque se haya realizado un proceso de paz y se perciba cierta dejación de armas, se mantiene un ambiente de incertidumbre por la continuidad de muertes de líderes sociales, mediadores de conflicto y la poca atención psicosocial a víctimas y familias de víctimas, lo que indica una presencia de injusticia social. Un entrevistado aduce: "El proceso de paz en Colombia en la actualidad tiende a fracasar porque no se han respetado del todo los acuerdos" (Comunicación personal, Pérez, 2017).

En cuanto al proceso de paz, en opinión de los docentes, este presenta las siguientes características desde las categorías que emergen en las entrevistas: dejación de armas, resentimiento, falta de atención en lo psicosocial, indiferencia al clamor de la familia, asesinato a líderes sociales mediadores de conflicto, poca participación ciudadana, esfuerzo loable, la paz utopía en Colombia, conexión armónica del corazón propio con el del otro, carácter constructivo, desmonte de estructura criminal que hacía mucho daño, dejación de armas y no de odios, justicia transicional que no satisface a todos, empezar aportar desde lo que soy para sanar heridas, perdonar, justicia y reparación, falta de plan de contingencia, no atención de secuelas en las víctimas, entre otras. Todas estas categorías que emergen se aglutinan en tres categorías abarcadoras: carácter constructivo de la paz, rasgos negativos y secuelas.

Por fin, después de 52 años de guerra en Colombia, se puede decir que lo acontecido es "el primer paso para alcanzar la paz, mas no la paz, y lo que se firmó fue un acuerdo de terminación de conflicto por uno de los actores, mas no la paz" (Comunicación personal, Lastre, 2017). Es indudable que la transformación individual afecta una colectividad y la paz individual irradia la colectividad. Por consiguiente, la terminación del conflicto quedó reglamentada en el Acto legislativo 01 de abril de 2017, donde se garantiza en el proceso de paz: entrega de armas, reparación, atención, no repetición.

En torno al carácter constructivo del proceso de paz desde la percepción de los maestros se logra ubicar en el contenido las siguientes categorías como: dejación de armas, esfuerzo loable, desmonte estructura criminal, justicia transicional (que no satisface), justicia y reparación (Figura 1).

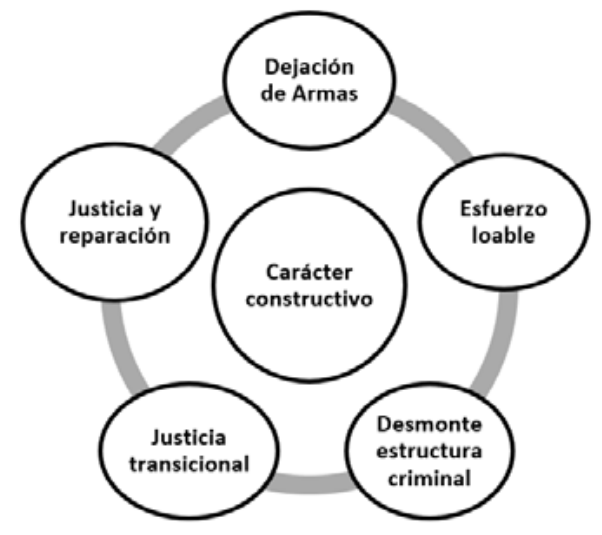

Figura 1. Carácter constructivo del proceso de paz

Fuente: elaboración propia.

El carácter constructivo de la paz desde la percepción de los maestros indica "un gran adelanto la dejación de armas, aunque no de odios y el esfuerzo del gobierno de turno" (Comunicación personal, Lora, 2017) Aunque no se vislumbre una paz duradera y se visibilicen totalmente los acuerdos divulgados en prensa durante todo el proceso de La Habana, Cuba, se infieren alcances favorables considerados como positivos y significativos por los maestros, los cuales expresan a manera de arbolada un ambiente fértil para la constitución de una paz real, aunque para algunos siga siendo una utopía.

Asimismo, se pudo agrupar alrededor de la categoría de rasgos negativos en el proceso de paz desde el imaginario del docente las subcategorías siguientes: asesinato a líderes sociales y mediadores de conflicto, falta de atención psicosocial, indiferencia al clamor de las familias víctimas $\mathrm{y}$ falta de un plan de contingencia (Figura 2).

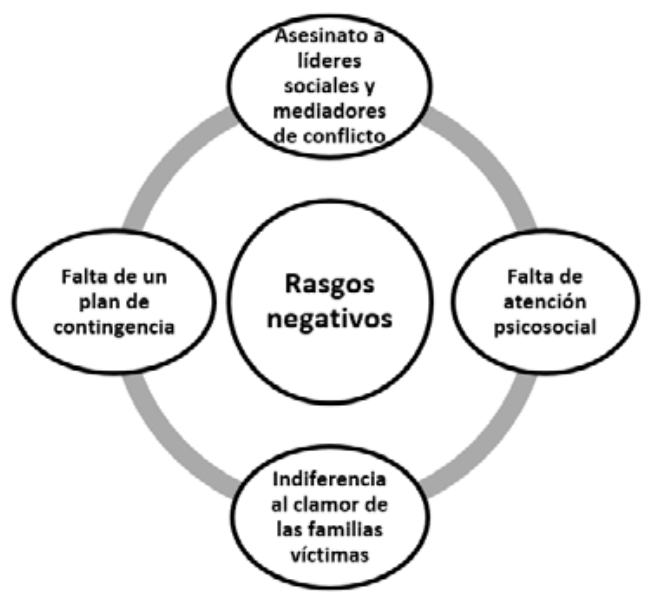

Figura 2. Rasgos negativos del proceso de paz

Fuente: elaboración propia. 
Es evidente en este aparte sobre rasgos negativos del proceso de paz la manera en la cual se aglutinan expresiones o ideas consideradas por los maestros como aspectos negativos que entorpecen el proceso de paz y crean un nimbo de desconfianza y miedo ante el devenir. Uno de ellos expresa que lo que se dio fue "un paso para alcanzar la paz y no la paz" (Comunicación personal, Lastre, 2017), puesto que aún se sigue vulnerando el derecho a la vida, a la libertad, a la paz. De igual forma, para algunos es necesario que se construya "un plan de contingencia que facilite los espacios y acuerdos necesarios para consolidar este proceso" (Comunicación personal, Galindo, 2017).

En cuanto a otro aspecto a considerar desde la percepción de los maestros en torno al proceso de paz, se logró agrupar una serie de aspectos que se enmarcan en la categoría secuelas, y se vinculan otras categorías que la sostienen producto de las voces de los maestros y están relacionadas con el odio y resentimiento tanto de víctima como de victimario. Atendiendo esto a un nivel (emocional); falta de perdón, ubicada desde la dimensión (espiritual); poca participación ciudadana en la apropiación de la paz, pues ellos consideran que un proceso de paz no es asunto de unos pocos, sino de toda la ciudadanía (Figura 3). Entonces, si se tiene un Estado que hace esfuerzos loables, las familias o el ciudadano común deben considerar la paz como un valor supremo.

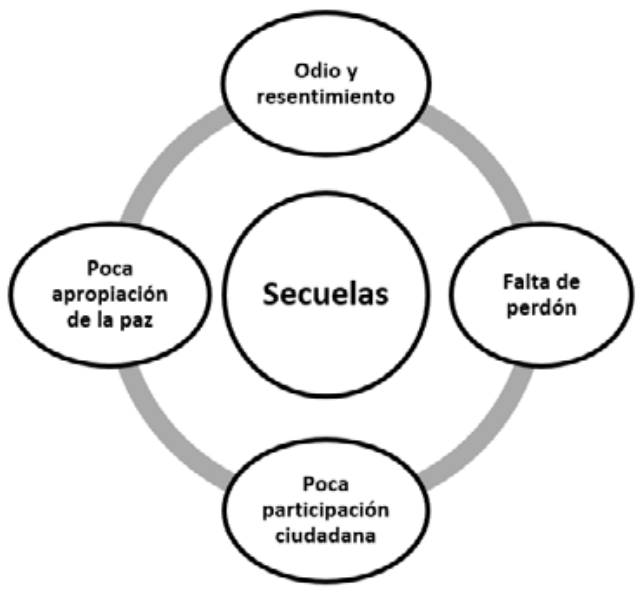

Figura 3. Secuelas

Fuente: elaboración propia.

Para ello, es prioridad atender un plan de salvaguardia para la paz que contribuye a preservar una cultura de paz; por consiguiente, la unión de todos los colombianos es importante y las garantías de la institucionalidad deben estar claras y monitoreadas mediante una ruta de atención especial a víctimas que facilite la atención y credibilidad en el proceso.

\section{Conclusión}

La paz es un proceso de construcción colectiva con responsabilidad social. De igual forma, solo es posible si se efectúan mejoras en las prácticas individuales y colectivas. Asimismo, desde las voces de maestros es multicolor, puesto que se presentan muchas percepciones atendiendo lo vivido y percibido. Se precisa en los maestros una visión objetiva y constructiva de la paz producto del análisis realizado a lecturas de la realidad. Por otro lado, en los estudiantes la visión de ella está orientada más a los anhelos y vivencias en armonía.

Existe un vínculo indeleble entre convivencia y paz, pues el contexto situacional posibilita la construcción de ideas, creencias y opiniones, tanto de forma individual como colectiva, y ellas direccionan el actuar del grupo, constituyéndose en patrón cultural.

Los imaginarios de paz se fundan y transforman a través del tiempo desde las sensaciones y percepciones del sujeto en la realidad que le corresponde vivir, lo que indica que son dinámicos y van cambiando con el pasar del tiempo.

El diálogo, como forma de abordaje del conflicto y como dimensión de la paz, debe estar presente en cada proceso de atención y reparación a víctimas del conflicto. La paz es portátil, cada ciudadano es portador de paz y violencia y decide desde la dimensión psicosocial donde se ubica.

La cultura es un factor determinante de la paz en la escuela, la familia y la sociedad en general. Esto significa que este concepto adquiere una connotación de complejidad; por consiguiente, se requiere hablar de una cultura de paz. La paz, como estado anhelado en las personas, es posible desde un conjunto de condiciones socioculturales que implican al Estado, escuela y familia como instituciones, trío perfecto para promover acciones mancomunadas sobre prácticas de paz.

En definitiva, la paz es el resultado de un cambio estructural en lo social, económico y político. Por tanto, solo es posible si el ciudadano se empodera y toma consciencia para actuar por convicción constituyéndose en una cultura de paz afianzada. Un docente expresaba empoderada... puesto que vivir juntos y en armonía no se consigue por decreto.

\section{Referencias}

Aigneren, M. (2002). La técnica de recolección de información mediante los grupos focales. Revista electrónica: la sociología en sus escenarios, (6)

Ávila, M. (2012). Educación para la paz: un reto educativo. REDHECS: Revista Electrónica de Humanidades, Educación y Comunicación Social, 8(14), 184-195.

Baltork, M., Mansoori, S. y Azad, Y. (2015). Content analysis of peace education as one component of global citizenship education in elementary textbooks. International Journal of Educational and Psychological Researches, 1(1), 28-35. 
Cengelci, T. y Gurdogan, O. (2016). Perception of peace in students' drawings. Eurasian Journal of Educational Research, (65), 181-198.

Chapela, L. (2013). Caja de herramientas en educación para la paz. Organización de las Naciones Unidas para la Educación, la Ciencia y la Cultura.

Congreso de Colombia (2017). Acto Legislativo 01 de 2017. http://es.presidencia.gov.co/normativa/normativa/ACTO\%20LEGISLATIVO $\% 20 \mathrm{~N} \% \mathrm{C} 2 \% \mathrm{~B} 0 \% 2001 \% 20 \mathrm{DE} \% 204 \% 20 \mathrm{DE} \% 20 \mathrm{ABRIL} \% 20$ DE\%202017.pdf

Constitución Política de Colombia (1991). Artículo 22.

Erreguerena, M. (2002). Cornelius Castoriadis: sus conceptos. Anuario 2001.

Freeman, M. (2013) Meaning making and understanding in focus groups: Affirming social and hermeneutic dialogue. En B. Dennis, L. Carspecken y P. Carspecken (Eds.). Qualitative Research: A Reader in Philosophy, Core Concepts, and Practice (pp. 131-148). Peter Lang Publishers.
Fuentes, L. (2018). Concepción de paz en estudiante de educación básica [manuscrito no publicado]. Universidad del Magdalena.

Fuentes, L., Pérez, L. y Medrano, Y. (2018). La escuela un espacio de convivencia. en F. Ramos, M., Rodríguez, H., Urzola, y D. Acosta, (Comp.). Formación Educativa en el contexto social y cultural. Volumen III, (pp. 37-52). Fondo Editorial UNERMB.

Palmer, R. (2002). ¿Qué es la hermenéutica? Teoría de la interpretación en Schleiermacher, Dilthey, Heidegger y Gadamer. Arco Libros.

Sagkal, A., Turnuklu, A. y Totan, T. (2012). Empathy for interpersonal peace: Effects of peace education on empathy skills. Kuram ve Uygulamada Egitim Bilimleri, 12(2), 1447-1460.

Organización de las Naciones Unidas para la Educación, la Ciencia y la Cultura [Unesco] (1995). Declaración de principios sobre la tolerancia: aprobada por la Conferencia General de la Unesco. http:// unesdoc.unesco.org/images/0015/001518/151830so.pdf

Organización de las Naciones Unidas para la Educación, la Ciencia y la Cultura [Unesco] (2011). Social and Human Sciences. 